Methods: mFSST administered on 22 patients undergoing rTKA. mFSST is performed by using tapes to make one horizontal and one vertical line like a cross to create 4 quadrants. Patients' performances were timed as patients were successfully stepping clockwise and counter-clockwise while avoid touching on tapes, turning their body or losing balance. Two trials performed and patients rested between trials and were encouraged to rest as often as they required to prevent fatigue. Results: ICC ${ }_{(2.1)}$ for mFSST was 0.83 . The standard error of measurement and MCID were 0.67 and 1.85 respectively ( $95 \%$. confidence level).

Conclusion: The mFSST has a good test-retest reliability in patients with rTKA. It is a reliable and responsive tool for measuring fall risk, dynamic balance and mobility. The mFSST is an excellent measure of gait variability, stepping in multipl directions and dynamic balance, also can easily identify real clinically important changes in patients with rTKA in simple environments and minimal equipment. References:

[1] Järvenpää J, Kettunen J, Miettinen H, Kröger H. The clinical outcome of revision knee replacement after unicompartmental knee arthroplasty versus primary total knee arthroplasty: 8-17 years follow-up study of 49 patients. International Orthopaedics 2010; 34: 649-653.

[2] Cumming RG, Klineberg RJ. Fall frequency and characteristics and the risk of hip fractures. Journal of the American Geriatrics Society 1994; 42: 774-778.

[3] Medell JL, Alexander NB. A clinical measure of maximal and rapid stepping in older women. The Journals of Gerontology Series A: Biological Sciences and Medical Sciences 2000; 55: M429-M433.

Disclosure of Interests: None declared

DOI: 10.1136/annrheumdis-2020-eular.5494

\section{AB0884 METABOLIC UNHEALTHY PHENOTYPE OF OBESITY IN PATIENTS WITH KNEE OSTEOARTHRITIS: THE EFFECTIVENESS OF ORLISTAT.}

E. Strebkova ${ }^{1}$, L. Alekseeva ${ }^{1} .{ }^{1}$ Federal State Budgetary Scientific Institution "Scientific Research Institute of Rheumatology named after V.A. Nasonova", Moscow, Russian Federation

Background: Obesity is an important socio-medical problem of mankind. Since the number of obese people in the world is increasing by about $1 \%$ per year, the immediate prospects do not look optimistic One of the important risks of the development and progression of osteoarthritis (OA) is the metabolically unhealthy phenotype of obesity, which is accompanied by a violation of lipid and carbohydrate metabolism, a violation of cytokine regulation. An important step in the treatment of patients with obesity and OA of the knee joints is weight loss.

Objectives: To evaluate the effectiveness of complex therapy of metabolic unhealthy phenotypes of obesity using orlistat (an intestinal lipase inhibitor) in the clinical manifestations of knee OA, dynamic markers of lipid and carbohydrate metabolism, dynamic CRP and leptin

Methods: The study included 50 female patients with knee OA Kellgren-Lawrence stage II-III and obesity (body mass index $(\mathrm{BMI})>30 \mathrm{~kg} / \mathrm{m}^{2}$ ), aged $45-65$ yo. Group 1 ( 25 patients) was administered orlistat at $120 \mathrm{mg}$ (1 capsule) 3 times a day for 6 month combined with low-calorie diet and therapeutic physical exercise. Group 2 (25 patients) was administered only life-modifying therapy for 6 month. The clinical parameters of the course of knee OA (WOMAC), an assessment of the quality of life (EQ-5D) were assessed at initially and after 6 months. Also initially and after 6 months a laboratory study of peripheral blood was performed glucose, HDL, LDL, TG, CRP, leptin, and the index of visceral obesity (IVO) was calculated.

Results: Initially, all patients had signs of a metabolically unhealthy phenotype of obesity: waist circumference (WC) $>88 \mathrm{~cm}$, high IVO, dyslipidemia, arterial hypertension, hyperleptinemia. After 6 months of complex therapy of obesity using orlistat in patients of group 1, a significant decrease in body weight by $10.07 \%(p<0.05)$, a decrease in WC by $8 \mathrm{~cm}(p<0.05)$, a significantly significant decrease were achieved IVO $(p<0.05)$, glucose $(p<0.05)$. Analysis of cytokines showed a significant decrease in leptin $(p<0.05)$ and CRP $(p<0.05)$ in patients with a weight loss of more than $10 \%$. The reduction in body weight in group 1 patients reduced pain by $52 \%(p<0.05)$, stiffness by $51 \%(p<0.05)$, improved joint functional failure by $51 \%(p<0.05)$ and improved quality life by $52 \%$ ( $p$ $<0.05$ ). In patients of group 2 , against the background of non-drug therapy of obesity, body weight decreased by $0.84 \%$ ( $p>0.05)$, slightly decreased WC ( $p>$ 0.05). Indicators of lipid metabolism, IVO, glucose, leptin remained unchanged ( $p>0.05$ ). The increase in CRP in patients of the 2nd group was determined by $24.54 \%(p<0.05)$.

Conclusion: The results of our study demonstrate the effectiveness of the use of a comprehensive drug regimen for the treatment of metabolically unhealthy phenotype of obesity for the clinical manifestations of osteoarthritis of the knee joints; it contributes to the improvement of metabolic parameters: a decrease in WC, lipid and carbohydrate metabolism, CRP and leptin. Thus, the use of complex therapy using orlistat in patients with osteoarthritis and obesity leads to a decrease in the metabolic activity of adipose tissue, helps to reduce cardiometabolic risk and to improve the clinical course of osteoarthritis.

Disclosure of Interests: None declared

DOI: 10.1136/annrheumdis-2020-eular.6292

\begin{tabular}{|l|l}
\hline AB0885 & EFFECTIVENESS AND SAFETY OF GLUCOSAMINE \\
& AND CHONDROITIN COMBINATION IN PATIENTS WITH \\
& KNEE AND HIP OSTEOARTHRITIS: INTERIM ANALYSIS \\
& RESULTS OF AN OBSERVATIONAL STUDY
\end{tabular}

K. Telyshev ${ }^{1}$, L. Alekseeva ${ }^{1}$, A. Lila ${ }^{2}$, A. Baranov ${ }^{3}$, E. Trofimov ${ }^{4}{ }^{1}$ Scientific Research Institute of Rheumatology named after V.A. Nasonova, Laboratory of Osteoarthritis, Moscow, Russian Federation; ${ }^{2}$ Scientific Research Institute of Rheumatology named after V.A. Nasonova, Moscow, Russian Federation; ${ }^{3}$ Yaroslavl State Medical University, Yaroslavl, Russian Federation; ${ }^{4}$ North-Western State Medical University named after I.I. Mechnikov, Saint Petersburg, Russian Federation

Background: Combined treatment with oral glucosamine hydrochloride $(\mathrm{GH})$ and chondroitin sulfate (CS) was shown to be efficient for pain relief and function improvement in osteoarthritis patients with moderate to severe knee pain $[1,2]$

Objectives: To investigate demographic and clinical characteristics, changes in pain, functions of daily living, quality of life and treatment satisfaction of patients with knee osteoarthritis (KOA) or hip osteoarthritis (HOA) receiving long-term treatment with oral $\mathrm{GH}$ and $\mathrm{CS}$ combination in routine clinical practice.

Methods: An open-label, multicenter, observational prospective study is being conducted in the Russian Federation. Patients of both sexes with KOA or HOA (Kellgren and Lawrence grades I-III) who receive GH 500 mg+CS 400 mg capsules three times a day for the first 3 weeks of treatment, then twice daily, are included in the study. The interim analysis has been conducted after the first 550 enrolled patients ( $50 \%$ of the total sample size) had completed the first follow-up visit (Week 16-24 after the start of treatment).

Results: The study group included 406 (73.8\%) patients with KOA and 144 $(26.2 \%)$ patients with HOA enrolled in 43 centers in Russia. The mean age of the patients was 61.1 years; most patients were women $(88.7 \%)$. The predominant risk factors for OA were non-genetic causes (excess weight, hormonal disorders, malformations of bones and joints, joint operations) (52.8\% of patients) exogenous risk factors (professional activity, trauma, sports) and hereditary diseases of bones and joints were reported in $15.5 \%$ and $4.1 \%$ of patients respectively.

Interim analysis showed clinically significant improvement in each of the KOOS and HOOS subscales at 4-6 months after the start of treatment. In patients with KOA, the mean score increase was 15.7 for the Pain subscale, 14.6 for the Quality of Life subscale, 13.8 for the Physical function (KOOS-PS), and 11.7 for the Symptoms subscale. The percentage of patients who rated the pain frequency as 'always' or 'daily' decreased from $60 \%$ to $25 \%$.

In patients with HOA, the mean score increase was 16.0 for the Pain subscale, 14.3 for the Quality of Life subscale, 16.1 for the Physical function (HOOS-PS), and 10.3 for the Symptoms subscale. The percentage of patients who rated the pain frequency as 'never' or 'monthly' increased from 34\% to $60 \%$.

Most patients $(89.1 \%)$ were receiving the medicinal product for $\geq 3$ months. Treatment-related AEs were reported in $16(3.0 \%)$ patients and mainly included gastrointestinal tract disorders (in $12(2.2 \%)$ patients)

Conclusion: The results obtained at 4-6 months after the start of treatment demonstrate clinically significant reduction of frequency and intensity of pain and other OA symptoms, as well as improvement of functions in daily living and quality of life in patients with KOA or HOA after the first course of treatment with $\mathrm{GH}+$ CS capsules. The majority of patients $(72.5 \%)$ were satisfied with the treatment The incidence of drug-related AEs was low, and the nature of AEs was consistent with known safety profile of $\mathrm{GH}$ and CS combination.

\section{References:}

[1] Clegg DO, Reda DJ, Harris CL, et al. Glucosamine, chondroitin sulfate, and the two in combination for painful knee osteoarthritis. N Engl J Med. 2006 Feb 23;354(8):795-808.

[2] Hochberg MC, Martel-Pelletier J, Monfort J, et al. Combined chondroitin sulfate and glucosamine for painful knee osteoarthritis: a multicentre, randomised, double-blind, non-inferiority trial versus celecoxib. Ann Rheum Dis. 2016 Jan;75(1):37-44

Disclosure of Interests: Kirill Telyshev: None declared, Ludmila Alekseeva Grant/research support from: Bayer, Alexander Lila: None declared, Andrey Baranov Grant/research support from: Bayer, Evgeny Trofimov Grant/research support from: Bayer

DOI: 10.1136/annrheumdis-2020-eular.3079 